\title{
Gender Differences in Cardiac Function Following Three- Month Administration of Tofogliflozin in Patients With Diabetes Mellitus
}

\author{
Toshihiro Higashikawa ${ }^{a}$, b, c , Tomohiko Ito ${ }^{a}$, Takurou Mizuno ${ }^{a}$, Keiichiro Ishigami ${ }^{a}$, \\ Masaru Kohori ${ }^{\mathrm{a}}$, Kunihiro Mae ${ }^{\mathrm{a}}$, Daisuke Usuda ${ }^{\mathrm{a}}$, Kento Takeshima ${ }^{\mathrm{a}}$, Susumu Takagia, \\ Toshihide Izumida a, Shinya Yamada ${ }^{a}$, Kengo Kuroki ${ }^{a}$, Ryusho Sangen ${ }^{a}$, Atsushi Saito ${ }^{\text {, }}$ \\ Masaharu Iguchia ${ }^{a}$ Yuji Kamasakia, Takeshi Nakahashib, Akihiro Fukuda ${ }^{a}$, \\ Tsugiyasu Kanda ${ }^{a}$, Masashi Okuro ${ }^{\mathrm{b}}$
}

\begin{abstract}
Background: Patients with type 2 diabetes mellitus (T2DM) are at increased risk for impairment in heart failure and diastolic relaxation while preserving ejection fraction (EF). Recently, several sodium glucose cotransporter-2 (SGLT2) inhibitors have demonstrated to decrease cardiovascular disease (CVD) events in elderly diabetic patients, although gender difference in the effect of SGLT2 inhibitors is unknown. The objective of the present study was to evaluate gender difference in the effect of tofogliflozin, one of the SGLT2 inhibitors, on CVD function in patients with diabetes mellitus.
\end{abstract}

Methods: This was a retrospective study. Patients received $20 \mathrm{mg}$ of tofogliflozin daily for 3 months. EF, ratio of early filling to atrial filling (E/A), a change in mitral inflow $\mathrm{E}$ and mitral $\mathrm{e}^{\prime}$ annular velocities (E/e'), left atrial dimension (LAD) and maximal diameter of inferior vena cava $\left(\mathrm{IVC}_{\max }\right)$, including various physiological parameters were measured between baseline, 1 month and 3 months after administration of tofogliflozin. Interaction between gender and time after administration was evaluated using mixed effect model.

Results: The results showed significant decrease in $\mathrm{E} / \mathrm{e}^{\prime}(\mathrm{P}<0.01)$ and significant interaction between time and gender in $\mathrm{E} / \mathrm{A}(\mathrm{P}<0.01)$, following administration of tofogliflozin for 3 months. EF was constantly higher significantly in women $(\mathrm{P}<0.01)$.

Conclusion: It is concluded that 3-month administration of tofogliflo-

Manuscript submitted July 1, 2020, accepted July 9, 2020

Published online July 22, 2020

aKanazawa Medical University Himi Municipal Hospital, 1130, Kurakawa, Himi, Toyama 935-8531, Japan

bDepartment of Geriatric Medicine, Kanazawa Medical University, Uchinada, Kahoku-gun, Ishikawa 920-0293, Japan

${ }^{\mathrm{c} C o r r e s p o n d i n g ~ A u t h o r: ~ T o s h i h i r o ~ H i g a s h i k a w a, ~ D e p a r t m e n t ~ o f ~ G e r i a t r i c ~}$ Medicine, Kanazawa Medical University Himi Municipal Hospital, 1130, Kurakawa, Himi, Toyama 935-8531, Japan. Email: himikmu@yahoo.co.jp

doi: https://doi.org/10.14740/jocmr4278 zin decreased $\mathrm{E} / \mathrm{e}^{\prime}$ with gender difference in $\mathrm{EF}$ and $\mathrm{E} / \mathrm{A}$.

Keywords: Tofogliflozin; Elderly; Diabetes mellitus; EF; E/A; E/e'

\section{Introduction}

Type 2 diabetes mellitus (T2DM) is one of the most serious causes of cardiovascular disease (CVD), including heart failure (HF) $[1,2]$. Sodium glucose cotransporter-2 (SGLT2) inhibitors have been emerged as a new class of antidiabetic drugs that inhibit glucose reabsorption in the renal proximal tubules followed by excretion of glucose into the urine [3]. Six SGTL2 inhibitors (ipragliflozin, dapagliflozin, tofogliflozin, canagliflozin, empagliflozin and luseogliflozin) have been currently available in Japan [4]. Several cardiovascular studies have found that SGLT2 inhibitors significantly reduced major adverse cardiovascular events, death and hospitalizations for HF during the treatment with canagliflozin, empagliflozin and dapagliflozin in T2DM patients with complication of CVD [5-8]. Furthermore, empagliflozin and canagliflozin have been known to decrease cardiovascular risk, including weight loss as well as lowering blood pressure [6, 8-10]. A prospective study has also found that treatment with dapagliflozin improved left ventricular (LV) diastolic functional parameters in T2DM patients with HF [11]. LV diastolic dysfunction has been known to have close correlation with HF $[12,13]$. Thus, the use of SGLT2 inhibitors which improves cardiovascular outcomes in patients with T2DM has been attracting attention. Tofogliflozin was found to improve LV dilatation and vascular endothelial function in Japanese patients with T2DM, presumably via elevation of ketone bodies induced by tofogliflozin which might contribute to a protective effect in LV diastolic dysfunction $[14,15]$. Recently, our study suggested that there were gender differences in the effect of tofogliflozin in Japanese elderly patients with T2DM using mixed effect model [16]. However, little is known concerning the gender difference of the effect, especially in elderly people. There is a tendency toward dias- 
tolic function aggravating in the elderly. From this view point, it is significant to assess diastolic function after administration of tofogliflozin. Tochiya et al also investigated the effect of tofogliflozin in patients with T2DM but almost all were men and gender difference remains unknown [15]. The objective of this study was to investigate gender differences in the effect of tofogliflozin on the cardiac functions, including LV diastolic function in patients with T2DM.

\section{Patients and Methods}

The present study was a retrospective study to investigate the effect of tofogliflozin, one of the SGLT2 inhibitors, on LV diastolic functional parameters in elderly patients with T2DM. This study included elderly patients with a diagnosis of T2DM who attended clinics at the Kanazawa Medical University Himi Municipal Hospital from April 2017 to March 2019. Elderly was defined as aged $\geq 65$ years. All patients received a single $20 \mathrm{mg}$ dose of tofogliflozin daily for 3 months. Patients with significant comorbid conditions were excluded. Demographic and baseline characteristics data were collected from patients' medical records. The data for patient characteristics were collected, gender (men/women), age (years), New York Heart Association (NYHA) classification, co-administered drugs, serum glycated hemoglobin (HbAlc), levels of hematocrit, brain natriuretic peptide (BNP), estimated glomerular filtration rate (eGFR), blood urea nitrogen (BUN), serum electrolyte (i.e. sodium, potassium and chloride) were measured at baseline, 1 month and 3 months.

Echocardiographic examination was performed with commercially available ultrasound systems, Hitachi Ultrasonic Diagnostic Apparatus Hivision Perius. Standard echocardiographic measurements were obtained in accordance with the current guidelines of the American Society of Echocardiography/European Association of Cardiovascular Imaging and the Guideline from Japanese Society of Echocardiography [13, 17, 18]. The maximal diameter of the inferior vena cava (IVCmax) was defined by the following procedure. Below the costal arch in parallel with the trunk and in the recumbent position before the inferior vein transitions to the right atrium at end expiration. The largest diameter was measured between 0.5 and $3 \mathrm{~cm}$ from the inflow to the right atrium in the IVC long axis crosssection at supine position. Maximum diameter was measured at expiration at the proximal hepatic vein junction. The left atrial dimension (LAD) was also measured. In parallel, ejection fraction $(\mathrm{EF})$ and the ratio of early filling to atrial filling (E/A) were obtained by the early diastolic (E) and atrial wave (A) velocities, and the E-wave deceleration time was measured by means of pulsed-wave Doppler recording from the apical four-chamber view. Spectral pulsed-wave Doppler-derived early diastolic velocity (e') was obtained by averaging the septal and lateral mitral annulus, and the mitral e' annular velocities $\left(\mathrm{E} / \mathrm{e}^{\prime}\right)$ were calculated.

To examine differences in characteristics of the patients between baseline and 3 months after tofogliflozin treatment, paired $t$-test was conducted for each variable, $\mathrm{HbAlc}$, body weight, systolic blood pressure, BNP, eGFR, BUN, serum
Table 1. Characteristics of the Patients

\begin{tabular}{|lll}
\hline Variable & Men $(\mathbf{N}=\mathbf{2 4})$ & Women $(\mathbf{N}=\mathbf{3 2})$ \\
\hline Age (years) & $80.2 \pm 6.4$ & $83.1 \pm 6.4$ \\
BMI & $23.1 \pm 3.3$ & $23.0 \pm 3.5$ \\
\hline NYHA I/II (III, IV: none) & $22 / 2$ & $28 / 2$ \\
\hline Co-administered drug & & \\
$\quad$ DPP-4 & 14 & 23 \\
BG & 7 & 11 \\
\hline SU & 5 & 3 \\
\hline Insulin & 0 & 0 \\
ARB & 6 & 6 \\
$\beta$-blocker & 5 & 4 \\
CCB & 10 & 17 \\
Diuretics & 4 & 13 \\
\hline
\end{tabular}

BMI: body mass index; NYHA: New York Heart Association; DPP-4: dipeptidyl peptidase-4; BG: biguanide; SU: sulphonylurea; ARB: angiotensin II receptor blocker; CCB: calcium channel blocker.

electrolytes, blood osmotic pressure, EF, E/A, E/e', LAD and diameter of IVC. In addition, to investigate the source of variation in EF, E/A, E/e', the fixed effect of treatment time period, gender and their interactions (time $\times$ gender) as well as the random effect of subjects were assessed using analysis of variance with a mixed effect model. All statistical analyses were twotailed, and $\mathrm{P}<0.05$ was regarded as statistically significant. The data were analyzed using the freely available EZR (Easy R) software (Saitama Medical Center, Jichi Medical University, Saitama, Japan) [19].

\section{Ethical considerations}

The study was conducted in accordance with the guidelines of the Declaration of Helsinki. The study was formally approved by the Clinical Research Ethics Committee of Kanazawa Medical University Himi Municipal Hospital (receipt no. 148).

\section{Results}

The baseline characteristics of 56 elderly patients with T2DM (men: 24, women: 32 ) are summarized in Table 1. The total number of patients included was 56. Their mean age was 82.0 \pm 6.5 (mean \pm standard deviation (SD)), with range of 67 96 years of age. NYHA I/II was 50/4. Co-administered drugs were dipeptidyl peptidase-4 (DPP-4) $(n=37)$, calcium channel blocker $(\mathrm{CCB})(\mathrm{n}=27)$, biguanide $(\mathrm{BG})(\mathrm{n}=18)$, diuretics $(\mathrm{n}=$ 16), etc. During the study period, myocardial infarction, stress cardiomyopathy and exacerbation of HF were not observed. Comparison of patient characteristics at baseline, 1 month and 3 months after administration of tofogliflozin is summarized in Table 2. HbA1c tended to decrease 3 months after administration of tofogliflozin from $7.4 \%$ at baseline to $6.7 \%$ at 3 months 
Table 2. Characteristics of Laboratory Test Results

\begin{tabular}{lllll}
\hline Variable & Baseline & 1 month & 3 months & P-value* \\
\hline HbAlc (\%) & $7.4 \pm 1.3$ & $7.0 \pm 1.0$ & $6.7 \pm 0.8$ & $<0.01$ \\
Body weight $(\mathrm{kg})$ & $54.6 \pm 10.9$ & $52.5 \pm 10.5$ & $51.8 \pm 10.1$ & $<0.01$ \\
Systolic blood pressure $(\mathrm{mm} \mathrm{Hg})$ & $138.4 \pm 24.5$ & $125.3 \pm 16.9$ & $128.0 \pm 20.1$ & $<0.01$ \\
Diastolic blood pressure $(\mathrm{mm} \mathrm{Hg})$ & $72.0 \pm 13.8$ & $70.5 \pm 11.5$ & $72.5 \pm 12.6$ & 0.81 \\
Hematocrit & $37.9 \pm 6.9$ & $38.4 \pm 5.9$ & $38.4 \pm 5.9$ & 0.3 \\
$\mathrm{BNP}(\mathrm{pg} / \mathrm{mL})$ & $222.8 \pm 378.5$ & $149.6 \pm 240.2$ & $186.8 \pm 270.6$ & 0.11 \\
eGFR & $60.5 \pm 23.2$ & $58.2 \pm 24.3$ & $59.3 \pm 28.4$ & 0.54 \\
$\mathrm{BUN}(\mathrm{mg} / \mathrm{dL})$ & $19.9 \pm 9.9$ & $19.7 \pm 7.2$ & $19.5 \pm 7.8$ & 0.74 \\
$\mathrm{Na}(\mathrm{mEq} / \mathrm{L})$ & $138.8 \pm 3.5$ & $139.5 \pm 3.0$ & $140.5 \pm 3.3$ & $<0.01$ \\
$\mathrm{~K}^{+}(\mathrm{mEq} / \mathrm{L})$ & $4.2 \pm 0.6$ & $4.2 \pm 0.5$ & $4.2 \pm 0.5$ & 0.78 \\
$\mathrm{Cl}$ (mEq/L) & $102.5 \pm 12.2$ & $104.4 \pm 5.0$ & $106.0 \pm 3.3$ & $<0.05$ \\
$\mathrm{Blood}$ osmotic pressure $(\mathrm{mOsm} / \mathrm{L})$ & $292.8 \pm 7.2$ & $292.6 \pm 7.3$ & $295.1 \pm 7.1$ & $<0.05$ \\
\hline
\end{tabular}

*Paired $t$-test compared to baseline with 3 months after administration. HbA1c: glycated hemoglobin; BNP: brain natriuretic peptide; eGFR: estimated glomerular filtration rate; BUN: blood urea nitrogen.

Table 3. Characteristics of Laboratory Test Results

\begin{tabular}{lllll}
\hline Variable & Baseline & 1 month & 3 months & P-value* \\
\hline EF & $64.5 \pm 8.9$ & $62.3 \pm 10.6$ & $63.3 \pm 9.3$ & 0.25 \\
E/A & $0.69 \pm 0.32$ & $0.60 \pm 0.18$ & $0.62 \pm 0.17$ & 0.31 \\
E/e' & $11.7 \pm 3.9$ & $9.9 \pm 2.9$ & $10.1 \pm 3.9$ & $<0.05$ \\
LAD $(\mathrm{mm})$ & $38.8 \pm 6.8$ & $37.4 \pm 6.7$ & $37.6 \pm 6.4$ & 0.19 \\
IVCmax $(\mathrm{mm})$ & $13.7 \pm 3.5$ & $13.5 \pm 3.7$ & $13.7 \pm 3.6$ & 0.61 \\
\hline
\end{tabular}

*Paired $t$-test compared to baseline with 3 months after administration. EF: ejection fraction; E/A: early filling/atrial filling; E/e': change in inflow E and mitral e' annular velocities; LAD: left atrial dimension; IVCmax: maximal diameter of inferior vena cava.

$(\mathrm{P}<0.01)$. Body weight significantly decreased from 54.6 to $51.8 \mathrm{~kg}(\mathrm{P}<0.01)$. Systolic blood pressures also showed significant decreases from 138.4 to $128.0 \mathrm{~mm} \mathrm{Hg}(\mathrm{P}<0.01)$, while diastolic blood pressure showed no significant changes from 72.0 to $72.5 \mathrm{~mm} \mathrm{Hg}(\mathrm{P}=0.81)$. Serum sodium and chloride significantly increased from 138.8 to $140.5 \mathrm{mEq} / \mathrm{L}$ and from 102.5 to $106.0 \mathrm{mEq} / \mathrm{L}$, respectively. Blood osmotic pressure showed significant increase from 292.8 to 295.1. No significant changes were observed on hematocrit, BNP, eGFR, BUN and serum potassium during the treatment with tofogliflozin.
Changes in echocardiographic endpoints are summarized in Table 3. After 3-month administration of tofogliflozin, EF and E/A showed no significant changes from 64.5 to 63.3 and 0.69 to 0.62 , respectively. The E/e' showed significant decrease from 11.7 to $10.1(\mathrm{P}<0.05)$. The LAD and IVCmax showed no significant change from 38.8 to $37.6 \mathrm{~mm}$ and from 13.7 to $13.7 \mathrm{~mm}$, respectively. The results of analysis of variance of EF with a mixed effect model are summarized in Table 4, showing that fixed effect (treatment time) was not significant, random effect (subjects) was significant $(\mathrm{P}<0.01)$ and gender

Table 4. Results of Two-Factor Mixed Effect Model to Evaluate Effects and Interaction of EF

\begin{tabular}{llllll} 
SOV & df & Sum sq. & Mean sq. & F & P-value \\
\hline Time & 2 & 125.1 & 62.5 & 2.4 & 7.5 \\
Subjects & 52 & $10,305.0$ & 198.2 & 9.1 & $<0.01$ \\
Gender & 1 & $1,796.5$ & $1,796.5$ & 1.5 & $<0.01$ \\
Time $\times$ gender & 2 & 81.1 & 40.5 & & 0.22 \\
Error & 105 & $2,760.2$ & 26.3 & & \\
Total & 162 & $15,067.8$ & & & \\
\hline
\end{tabular}

Analysis of variance table for the mixed effects model. EF: ejection fraction; SOV: source of variance; df: degree of freedom; Sum sq.: sum of squares; Mean sq.: mean squares; F: F statistic. 


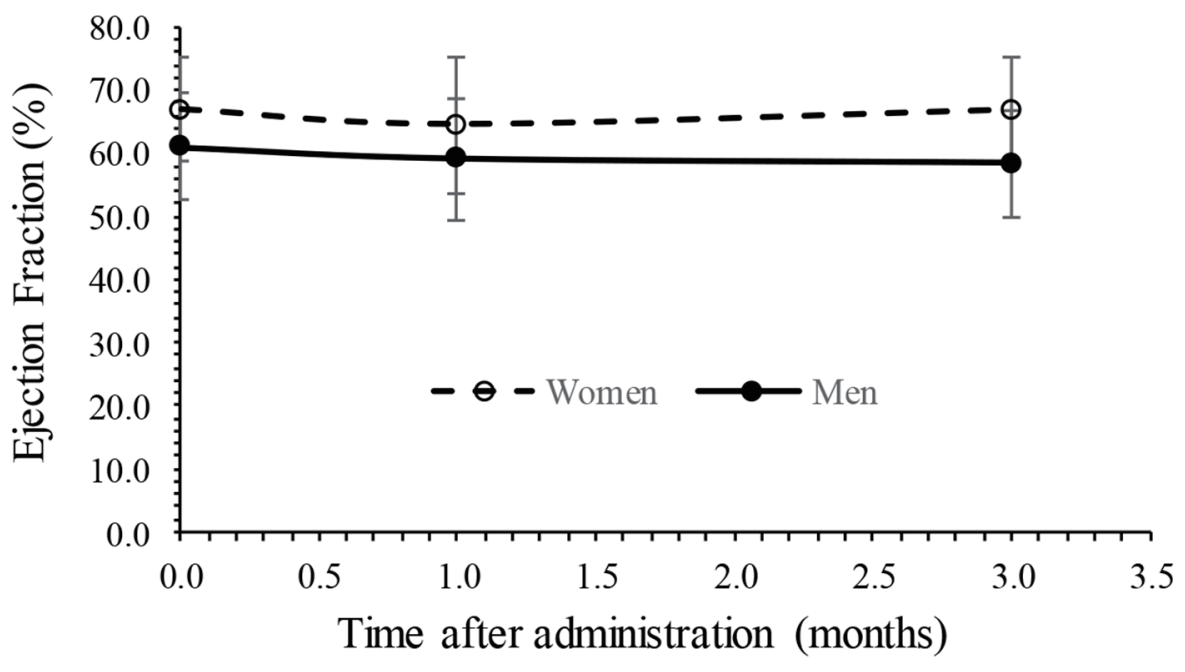

Figure 1. Interaction plot in EF between time and gender. EF: ejection fraction.

Table 5. Results of Two-Factor Mixed Effect Model to Evaluate Effects and Interaction of E/A

\begin{tabular}{llllll} 
SOV & df & Sum sq. & Mean sq. & F & P-value \\
\hline Time & 2 & 0.04 & 0.02 & 2.7 & 8.9 \\
Subjects & 35 & 2.6 & 0.1 & 0.07 & $<0.01$ \\
Gender & 1 & 0.05 & 0.05 & 7.2 & 0.40 \\
Time $\times$ gender & 2 & 0.12 & 0.06 & & $<0.01$ \\
Error & 105 & 0.87 & 0.01 & & \\
Total & 162 & 3.7 & & & \\
\hline
\end{tabular}

Analysis of variance table for the mixed effects model. E/A: early filling/atrial filling; SOV: source of variance; Sum sq.: sum of squares; df: degree of freedom; Mean sq.: mean squares; F: F statistic.

effect was also significant $(\mathrm{P}<0.01)$, whereas the interaction (time $\times$ gender) was not significant. No interaction can be observed because the time trend of EF was parallel between men and women as shown in Figure 1. The results of analysis of variance of E/A with a mixed effect model are also summarized in Table 5, showing that fixed effect (treatment time) and gender was not significant, whereas random effect (subjects) and the interaction (time $\times$ gender) were significant $(\mathrm{P}<0.01)$.

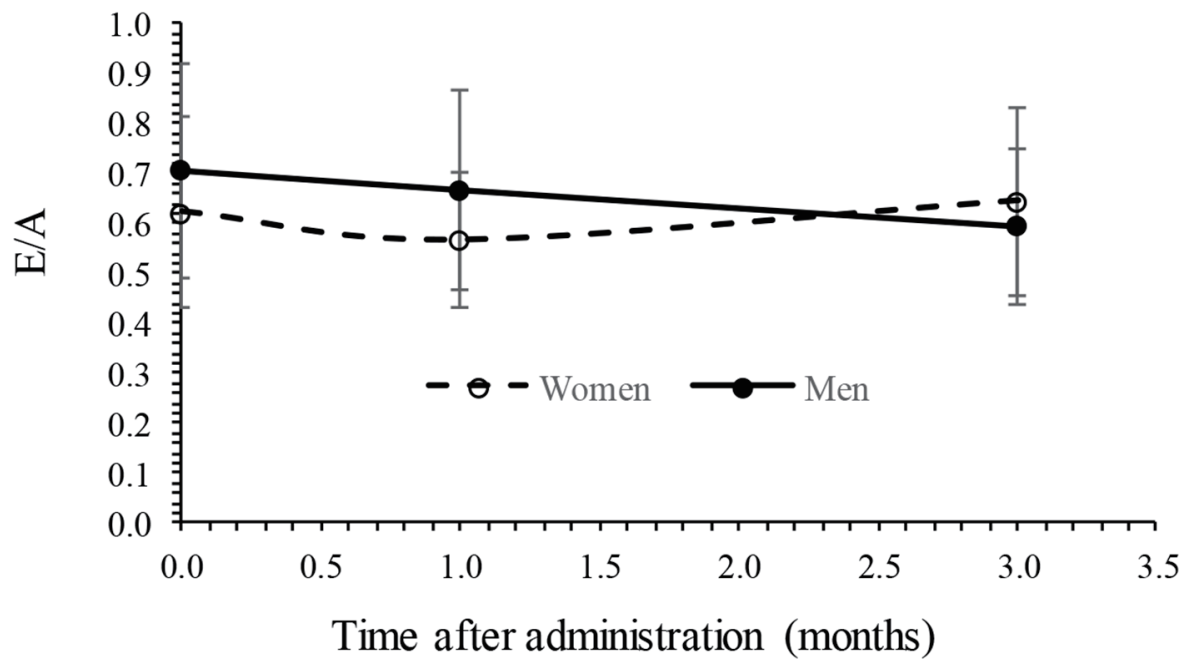

Figure 2. Interaction plot in E/A between time and gender. E/A: early filling/atrial filling. 
Table 6. Results of two-factor mixed effect model to evaluate effects and interaction of E/e'

\begin{tabular}{|c|c|c|c|c|c|}
\hline SOV & df & Sum sq. & Mean sq. & $\mathbf{F}$ & P-value \\
\hline Time & 2 & 60.9 & 30.5 & 11.5 & $<0.01$ \\
\hline Subjects & 52 & 1012.9 & 19.5 & 7.4 & $<0.01$ \\
\hline Time $\times$ gender & 2 & 11.1 & 5.6 & 2.1 & 0.13 \\
\hline Error & 105 & 277.3 & 2.6 & & \\
\hline
\end{tabular}

Analysis of variance table for the mixed effects model. E/e': change in inflow E and mitral e' annular velocities; SOV: source of variance; Sum sq.: sum of squares; df: degree of freedom; Mean sq.: mean squares; F: F statistic.

The interaction can be observed because the time trends of $\mathrm{E} / \mathrm{A}$ were crossed between men and women as shown in Figure 2. The results of analysis of variance of E/e' with a mixed effect model were also summarized in Table 6, showing that fixed effect (treatment time) and random effect (subjects) were significant $(\mathrm{P}<0.05)$, whereas gender and the interaction (time $\times$ gender) were not significant. Figure 3 shows an interaction plot where women are more likely to decrease than men. The interaction was not significant $(\mathrm{P}=0.13)$.

\section{Discussion}

The present study found that LV diastolic function as measured by E/e' for patients with T2DM had significantly improved 3 months after administration of tofogliflozin in elderly patients with T2DM, consistent with the previous results [14-16]. Other cardiac functional parameters such as diameter of LAD and IVCmax did not change 3 months after administration of tofogliflozin. The results also suggested that body weight and systolic blood pressure significantly decreased, also consistent with the previous results [20]. The results of analysis of variance of cardiac parameters $\mathrm{EF}, \mathrm{E} / \mathrm{A}$ and $\mathrm{E} / \mathrm{e}^{\prime}$ with a mixed effect model found that the level of EF was higher in women than men, interaction (time $\times$ gender) was significant in $\mathrm{E} / \mathrm{A}$, and time (fixed effect) was significant in E/e'. It has already been known previously that preserved EF tends to be higher in women than men especially in elderly, consistent with the present results [21, 22]. Men are more often contractile disorders in patients with obesity, hypertension, diabetes mellitus, anemia and chronic kidney disease than in women, and in women, estrogen, a women hormone, acts protectively on the myocardium, suggesting the possibility of preserving the LV diastolic function [23, 24].

After menopause, the absence of estrogen causes distension. Hemodynamic effects, such as reductions in blood pressure and intravascular volume, involving osmotic diuresis, may provide a more plausible explanation. Metabolic effects, such as cardiac fuel energetics, and hormonal effects, such as increased glucagon release, may also be included in the mechanism [25]. T2DM is one of the most crucial comorbidities associated with CVD that attributes to end organ damage. T2DM also increases the risk for developing HF [26] and its associated complications, including death [27]. SGLT2 inhibitors have been known to improve glycemia and promote weight loss and body fat mass reduction in humans and animals with diabetes, in part, due to caloric loss by high excretion of urinary glucose and an enhancement of oxidative metabolism from

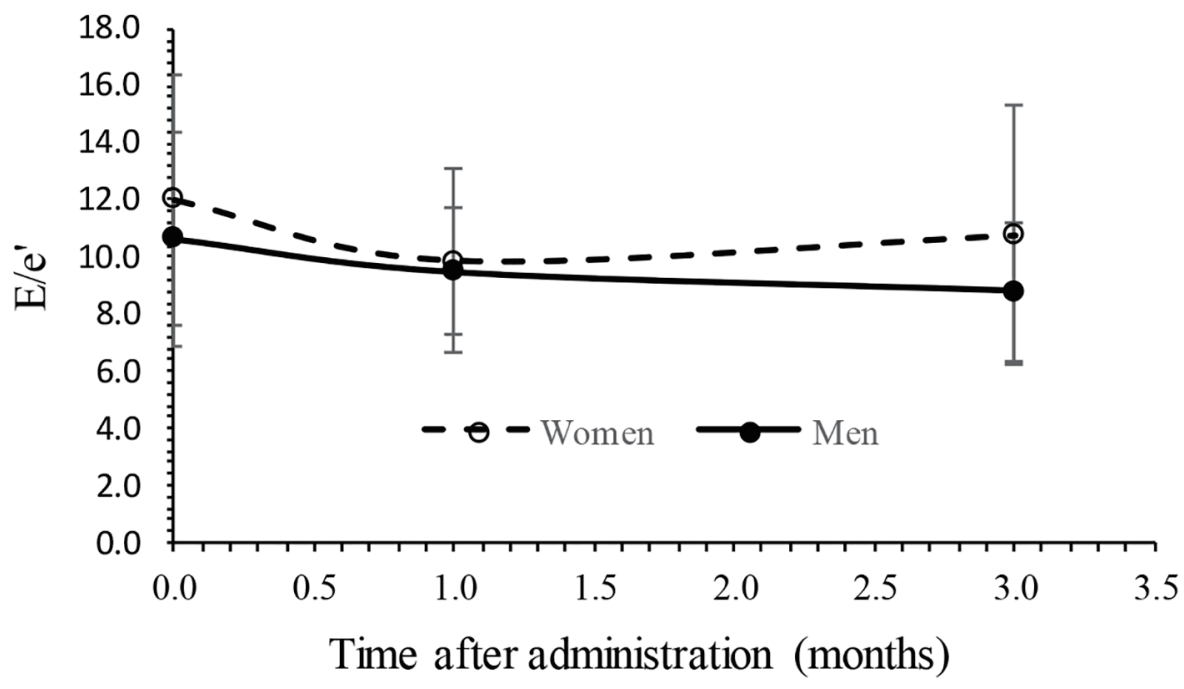

Figure 3. Interaction plot in E/e' between time and gender. E/e': change in inflow E and mitral e' annular velocities. 
carbohydrate to fatty acid $[28,29]$. The effect of tofogliflozin on cardiac function has been known to increase EF from $48 \%$ to $61 \%$, in parallel with increase of ketone bodies (betahydroxybutyrate and acetoacetate) levels in a T2DM case [30]. Although we did not measure ketone bodies, the mechanism of the effect may underlie some metabolic shift. Tofogliflozin has been known to be a most selective SGLT2 inhibitor. Compared to SGLT1, the selectivity of tofogliflozin is highest, approximately 3,000-fold [31]. SGLT2 transporter is located mostly in the kidney, whereas SGLT1 is located in various organs, i.e. small intestine, kidney, parotid glands, submandibular glands and in the heart [32]. These results suggest that the effect of SGLT2 inhibitors on cardiac functions commonly observed by both tofogliflozin and canagliflozin may not be mediated via SGLT1. LV diastolic dysfunction is the most frequently observed early LV functional abnormality in patients with T2DM [33]. The LV diastolic dysfunction is thought to be due to the underlying pathophysiological abnormality of cardiac function, and thus its assessment plays an important role for diagnosis of patients with HF. The LV diastolic dysfunction is known to be independently relevant to outcomes in patients with HF $[12,13,34]$. SGLT2 inhibitors have a multimodal effect on cardiac function including improvement in endothelial dysfunction and aortic stiffness [35]. Short-term treatment with empagliflozin is known to reduce LV diastolic function as assessed in terms of e' [36]. The effects of canagliflozin on LV diastolic function as assessed by E/e' and left ventricular mass index (LVMI) had significantly improved by 3 months treatment of canagliflozin while maintaining EF [37]. A previous study also showed that 6-month treatment with dapagliflozin improved LV diastolic functional parameters including E/e', left atrial volume index (LAVI) and LVMI [11]. Although the precise mechanism of the effect of SGLT2 inhibitors on LV diastolic function remains uncertain, significant correlation was reported between $\mathrm{E} / \mathrm{e}^{\prime}$ and pulmonary capillary wedge pressure $[38,39]$, indicating that decrease of $\mathrm{E} / \mathrm{e}^{\prime}$ would be beneficial in T2DM patients with HF.

The present study also showed significant decrease in systolic blood pressure during 3-month treatment with tofogliflozin, consistent with the Japanese post-marketing study [20]. Reductions in blood pressure are known to correlate with the amelioration of albuminuria in patients with T2DM [40]. Several clinical trials have also shown that SGLT2 inhibitors exert blood pressure-lowering effects through their diuretic actions, which may partially contribute to the amelioration of albuminuria in diabetic patients [41]. On the other hand, in a rodent study high-dose ipragliflozin reduced the LV chamber size while maintaining stably in blood pressure [42]. The effect of SGLT2 inhibitor on blood pressure still remains controversial, which may be partially caused by the time at which blood pressure was measured. Tofogliflozin has also been known to improve insulin resistance in both animals and humans [43-45].

\section{Study limitations}

The results of our study should be considered in the context of several potential limitations. First, due to the observational nature of the study, and despite robust statistical techniques, a possibility of residual, unmeasured confounding cannot be excluded. Specifically, certain patient and physician factors may not be adequately captured. Second, the present study evaluated only 3 months of drug treatment period, which could be insufficient to detect change in physiological parameters. Longer-term follow-up will be needed to clarify the effects of tofogliflozin on various physiological parameters. Second, the present study lacks control groups, thus existence of underlying contributors (exercise, diet, restricting smoking and alcohol, etc.) could affect the outcome of this study.

\section{Conclusion}

The present study concluded that 3-month administration of tofogliflozin decreased E/e' with gender difference in EF and $\mathrm{E} / \mathrm{A}$, under preserving IVC, renal function and electrolyte balance. Tofogliflozin could improve LV diastolic function in elderly patients with T2DM. SGLT2 inhibition may emerge as an effective and safe adjunctive therapy for HF that promotes hemodynamic stability and helps correct volume overload, while avoiding the risks of volume depletion, independent of effects on hyperglycemia. Nevertheless, the mechanisms responsible for the acute cardioprotective effects of SGLT2 inhibition, such as sodium and water homeostasis and plasma volume regulation remain unknown in patients with T2DM and in those with HF. Further studies will be expected to elucidate these mechanisms.

\section{Acknowledgments}

We thank Sonomi Suzaki, Mayumi Terashita, Saori Nishioka, Takuya Saeki and Misato Ueno for preparation of the manuscript.

\section{Financial Disclosure}

The present study was financially supported by Kowa K.K.

\section{Conflict of Interest}

None to declare.

\section{Informed Consent}

Informed consents were obtained from all participants.

\section{Author Contributions}

Toshihiro Higashikawa and Tomohiko Ito have made data curation. Toshihiro Higashikawa, Takurou Mizuno, Keiichiro Ishigami, Takurou Mizuno and Kento Takashima designed 
the research. Toshihiro Higashikawa conducted data analysis. Toshihiro Higashikawa acquired funding. Toshihiro Higashikawa, Masaru Kohori, Kunihiro Mae and Daisuke Usuda conducted investigation of medical records. Toshihiro Higashikawa, Susumu Takagi, Kento Takeshima, Toshihide Izumida, Shinya Yamada, Kengo Kuroki, Ryusho Sangen and Keito Takashima contributed to methodology. Atsushi Saito, Masaharu Iguchi, Yuji Kasamaki, Akihiro Fukuda, Takeshi Nakahashi, Takashi Nakahashi, Tsugiyasu Kanda and Masashi Okuro supervised this research. Toshihiro Higashikawa wrote original draft of this manuscript. All authors read and approved the final manuscript.

\section{Data Availability}

The data supporting the findings of this study are available from the corresponding author upon reasonable request.

\section{Abbreviations}

T2DM: type 2 diabetes mellitus; EF: ejection fraction; E/A: early filling/atrial filling; E/e': change in inflow $\mathrm{E}$ and mitral $\mathrm{e}^{\prime}$ annular velocities; LAD: left atrial dimension; IVCmax: maximal diameter of inferior vena cava

\section{References}

1. Kannel WB, McGee DL. Diabetes and cardiovascular disease. The Framingham study. JAMA. 1979;241(19):20352038.

2. Vazquez-Benitez G, Desai JR, Xu S, Goodrich GK, Schroeder EB, Nichols GA, Segal J, et al. Preventable major cardiovascular events associated with uncontrolled glucose, blood pressure, and lipids and active smoking in adults with diabetes with and without cardiovascular disease: a contemporary analysis. Diabetes Care. 2015;38(5):905-912.

3. Nair S, Wilding JP. Sodium glucose cotransporter 2 inhibitors as a new treatment for diabetes mellitus. J Clin Endocrinol Metab. 2010;95(1):34-42.

4. Tahara A, Takasu T, Yokono M, Imamura M, Kurosaki E. Characterization and comparison of sodium-glucose cotransporter 2 inhibitors in pharmacokinetics, pharmacodynamics, and pharmacologic effects. J Pharmacol Sci. 2016;130(3):159-169.

5. Mahaffey KW, Neal B, Perkovic V, de Zeeuw D, Fulcher G, Erondu N, Shaw W, et al. Canagliflozin for primary and secondary prevention of cardiovascular events: results from the CANVAS Program (Canagliflozin Cardiovascular Assessment Study). Circulation. 2018;137(4):323334.

6. Neal B, Perkovic V, Mahaffey KW, de Zeeuw D, Fulcher G, Erondu N, Shaw W, et al. Canagliflozin and cardiovascular and renal events in type 2 diabetes. N Engl J Med. 2017;377(7):644-657.
7. Wiviott SD, Raz I, Bonaca MP, Mosenzon O, Kato ET, Cahn A, Silverman MG, et al. Dapagliflozin and cardiovascular outcomes in type 2 diabetes. N Engl J Med. 2019;380(4):347-357.

8. Zinman B, Wanner C, Lachin JM, Fitchett D, Bluhmki E, Hantel S, Mattheus M, et al. Empagliflozin, cardiovascular outcomes, and mortality in type 2 diabetes. N Engl J Med. 2015;373(22):2117-2128.

9. Haring HU, Merker L, Seewaldt-Becker E, Weimer M, Meinicke T, Woerle HJ, Broedl UC, et al. Empagliflozin as add-on to metformin plus sulfonylurea in patients with type 2 diabetes: a 24-week, randomized, double-blind, placebo-controlled trial. Diabetes Care. 2013;36(11):3396-3404.

10. Rosenstock J, Jelaska A, Frappin G, Salsali A, Kim G, Woerle HJ, Broedl UC, et al. Improved glucose control with weight loss, lower insulin doses, and no increased hypoglycemia with empagliflozin added to titrated multiple daily injections of insulin in obese inadequately controlled type 2 diabetes. Diabetes Care. 2014;37(7):18151823.

11. Soga F, Tanaka H, Tatsumi K, Mochizuki Y, Sano H, Toki $\mathrm{H}$, Matsumoto $\mathrm{K}$, et al. Impact of dapagliflozin on left ventricular diastolic function of patients with type 2 diabetic mellitus with chronic heart failure. Cardiovasc Diabetol. 2018;17(1):132.

12. Andersen OS, Smiseth OA, Dokainish H, Abudiab MM, Schutt RC, Kumar A, Sato K, et al. Estimating Left Ventricular Filling Pressure by Echocardiography. J Am Coll Cardiol. 2017;69(15):1937-1948.

13. Ponikowski P, Voors AA, Anker SD, Bueno H, Cleland JGF, Coats AJS, Falk V, et al. 2016 ESC Guidelines for the diagnosis and treatment of acute and chronic heart failure: The Task Force for the diagnosis and treatment of acute and chronic heart failure of the European Society of Cardiology (ESC)Developed with the special contribution of the Heart Failure Association (HFA) of the ESC. Eur Heart J. 2016;37(27):2129-2200.

14. Otagaki M, Matsumura K, Kin H, Fujii K, Shibutani H, Matsumoto H, Takahashi H, et al. Effect of Tofogliflozin on Systolic and Diastolic Cardiac Function in Type 2 Diabetic Patients. Cardiovasc Drugs Ther. 2019;33(4):435442.

15. Tochiya M, Makino H, Tamanaha T, Matsuo M, Hishida A, Koezuka R, Ohata Y, et al. Effect of tofogliflozin on cardiac and vascular endothelial function in patients with type 2 diabetes and heart diseases: A pilot study. J Diabetes Investig. 2020;11(2):400-404.

16. Higashikawa T, Ito T, Mizuno T, Ishigami K, Kohori M, Mae K, Usuda D, et al. Effects of tofogliflozin on cardiac function in elderly patients with diabetes mellitus. J Clin Med Res. 2020;12(3):165-171.

17. Daimon M, Akaishi M, Asanuma T, Hashimoto S, Izumi C, Iwanaga S, Kawai H, et al. Guideline from Japanese Society of Echocardiography: 2018 focused update incorporated into Guidance for the Management and Maintenance of Echocardiography Equipment. J Echocardiogr. 2018;16(1):1-5.

18. Ommen SR, Nishimura RA, Appleton CP, Miller FA, Oh 
JK, Redfield MM, Tajik AJ. Clinical utility of Doppler echocardiography and tissue Doppler imaging in the estimation of left ventricular filling pressures: A comparative simultaneous Doppler-catheterization study. Circulation. 2000;102(15):1788-1794.

19. Kanda Y. Investigation of the freely available easy-touse software 'EZR' for medical statistics. Bone Marrow Transplant. 2013;48(3):452-458.

20. Utsunomiya K, Shimmoto N, Senda M, Kurihara Y, Gunji R, Fujii S, Kakiuchi S, et al. Safety and effectiveness of tofogliflozin in elderly Japanese patients with type 2 diabetes mellitus: A post-marketing study (J-STEP/EL Study). J Diabetes Investig. 2017;8(6):766-775.

21. Nakada Y, Kawakami R, Nakano T, Takitsume A, Nakagawa $H$, Ueda T, Nishida $T$, et al. Sex differences in clinical characteristics and long-term outcome in acute decompensated heart failure patients with preserved and reduced ejection fraction. Am J Physiol Heart Circ Physiol. 2016;310(7):H813-820.

22. Ozlek B, Ozlek E, Kahraman S, Tekinalp M, Zencirkiran Agus H, Celik O, Cil C, et al. Gender disparities in heart failure with mid-range and preserved ejection fraction: Results from APOLLON study. Anatol J Cardiol. 2019;21(5):242-252.

23. Keller KM, Howlett SE. Sex Differences in the Biology and Pathology of the Aging Heart. Can J Cardiol. 2016;32(9):1065-1073.

24. Rusinaru D, Tribouilloy C, Berry C, Richards AM, Whalley GA, Earle N, Poppe KK, et al. Relationship of serum sodium concentration to mortality in a wide spectrum of heart failure patients with preserved and with reduced ejection fraction: an individual patient data meta-analysis(dagger): Meta-Analysis Global Group in Chronic heart failure (MAGGIC). Eur J Heart Fail. 2012;14(10):1139-1146.

25. Staels B. Cardiovascular Protection by Sodium Glucose Cotransporter 2 Inhibitors: Potential Mechanisms. Am J Med. 2017;130(6S):S30-S39.

26. Mentz RJ, Kelly JP, von Lueder TG, Voors AA, Lam CS, Cowie MR, Kjeldsen K, et al. Noncardiac comorbidities in heart failure with reduced versus preserved ejection fraction. J Am Coll Cardiol. 2014;64(21):2281-2293.

27. Kannel WB, Hjortland M, Castelli WP. Role of diabetes in congestive heart failure: the Framingham study. Am J Cardiol. 1974;34(1):29-34.

28. Ferrannini E, Ramos SJ, Salsali A, Tang W, List JF. Dapagliflozin monotherapy in type 2 diabetic patients with inadequate glycemic control by diet and exercise: a randomized, double-blind, placebo-controlled, phase 3 trial. Diabetes Care. 2010;33(10):2217-2224.

29. Nishimura R, Tanaka Y, Koiwai K, Inoue K, Hach T, Salsali A, Lund SS, et al. Effect of empagliflozin monotherapy on postprandial glucose and 24-hour glucose variability in Japanese patients with type 2 diabetes mellitus: a randomized, double-blind, placebo-controlled, 4-week study. Cardiovasc Diabetol. 2015;14:11.

30. Kutoh E, Hayashi J. Effect of canagliflozin on heart function involving ketone bodies in patients with type 2 diabetes. Drug Res (Stuttg). 2019;69(5):297-300.
31. Abdul-Ghani MA, DeFronzo RA, Norton L. Novel hypothesis to explain why SGLT2 inhibitors inhibit only $30-50 \%$ of filtered glucose load in humans. Diabetes. 2013;62(10):3324-3328.

32. Madunic IV, Breljak D, Karaica D, Koepsell H, Sabolic I. Expression profiling and immunolocalization of $\mathrm{Na}(+)-$ D-glucose-cotransporter 1 in mice employing knockout mice as specificity control indicate novel locations and differences between mice and rats. Pflugers Arch. 2017;469(12):1545-1565.

33. Fang ZY, Prins JB, Marwick TH. Diabetic cardiomyopathy: evidence, mechanisms, and therapeutic implications. Endocr Rev. 2004;25(4):543-567.

34. Henning RJ. Diagnosis and treatment of heart failure with preserved left ventricular ejection fraction. World J Cardiol. 2020;12(1):7-25.

35. Solini A, Giannini L, Seghieri M, Vitolo E, Taddei S, Ghiadoni L, Bruno RM. Dapagliflozin acutely improves endothelial dysfunction, reduces aortic stiffness and renal resistive index in type 2 diabetic patients: a pilot study. Cardiovasc Diabetol. 2017;16(1):138.

36. Verma S, Garg A, Yan AT, Gupta AK, Al-Omran M, Sabongui A, Teoh $\mathrm{H}$, et al. Effect of empagliffozin on left ventricular mass and diastolic function in individuals with diabetes: an important clue to the EMPA-REG OUTCOME trial? Diabetes Care. 2016;39(12):e212-e213.

37. Matsutani D, Sakamoto M, Kayama Y, Takeda N, Horiuchi R, Utsunomiya K. Effect of canagliflozin on left ventricular diastolic function in patients with type 2 diabetes. Cardiovasc Diabetol. 2018;17(1):73.

38. Lam CS, Roger VL, Rodeheffer RJ, Borlaug BA, Enders FT, Redfield MM. Pulmonary hypertension in heart failure with preserved ejection fraction: a community-based study. J Am Coll Cardiol. 2009;53(13):1119-1126.

39. Matsushita K, Minamishima T, Goda A, Ishiguro H, Kosho H, Sakata K, Satoh T, et al. Comparison of the reliability of $\mathrm{E} / \mathrm{E}^{\prime}$ to estimate pulmonary capillary wedge pressure in heart failure patients with preserved ejection fraction versus those with reduced ejection fraction. Int J Cardiovasc Imaging. 2015;31(8):1497-1502.

40. Emdin CA, Rahimi K, Neal B, Callender T, Perkovic V, Patel A. Blood pressure lowering in type 2 diabetes: a systematic review and meta-analysis. JAMA. 2015;313(6):603-615.

41. Baker WL, Smyth LR, Riche DM, Bourret EM, Chamberlin KW, White WB. Effects of sodium-glucose co-transporter 2 inhibitors on blood pressure: a systematic review and meta-analysis. J Am Soc Hypertens. 2014;8(4):262275 e269.

42. Kamezaki M, Kusaba T, Komaki K, Fushimura Y, Watanabe N, Ikeda K, Kitani T, et al. Comprehensive renoprotective effects of ipragliflozin on early diabetic nephropathy in mice. Sci Rep. 2018;8(1):4029.

43. Kubota T, Kubota N, Kumagai H, Yamaguchi S, Kozono $\mathrm{H}$, Takahashi $\mathrm{T}$, Inoue $\mathrm{M}$, et al. Impaired insulin signaling in endothelial cells reduces insulin-induced glucose uptake by skeletal muscle. Cell Metab. 2011;13(3):294-307.

44. Obata A, Kubota N, Kubota T, Iwamoto M, Sato H, Sakurai Y, Takamoto I, et al. Tofogliflozin improves 
insulin resistance in skeletal muscle and accelerates lipolysis in adipose tissue in male mice. Endocrinology. 2016;157(3):1029-1042.

45. Tobe K, Suganami H, Kaku K. Sodium-glucose cotrans- porter 2 inhibitor, tofogliflozin, shows better improvements of blood glucose and insulin secretion in patients with high insulin levels at baseline. J Diabetes Investig. 2018;9(4):862-869. 